\title{
Overexpression of neutrophil gelatinase-associated lipocalin and its receptor in colorectal carcinoma: Significant correlation with cell differentiation and tumour invasion
}

\author{
ZHUO LV ${ }^{1}$, LI-YAN XU², ZHONG-YING SHEN ${ }^{2}$, FA-REN ZHANG ${ }^{1}$, XIU-E XU ${ }^{2}$ and EN-MIN LI ${ }^{1}$ \\ ${ }^{1}$ Department of Biochemistry and Molecular Biology, ${ }^{2}$ The Institute of Oncologic Pathology, \\ Medical College of Shantou University, Shantou 515041, P.R. China
}

Received February 23, 2009; Accepted April 30, 2009

DOI: 10.3892/ol_00000000

\begin{abstract}
Neutrophil gelatinase-associated lipocalin (NGAL), a member of the lipocalin family, is related to imflammation and tumour. Recently, a specific cell-surface receptor $(24 \mathrm{p} 3 \mathrm{R} /$ NGALR) for lipocalin 24p3 was reported. However, the characteristics of NGALR expression in colorectal carcinoma (CRC) are not known. The objectives of this study were to investigate the expression of NGAL and NGALR in CRC specimens, and determine any relationship between the expression of these proteins and tumour progression. In the present study, CRC specimens of 102 patients were obtained, and the expression of NGAL, NGALR, ferritin and Ki67 was analyzed in paraffin sections by immunohistochemistry. Statistical analyses of the data collected were performed with SPSS software. We found that the cytoplasmic staining of NGAL, NGALR and ferritin, as well as the nuclear staining of Ki67 were significantly upregulated in CRC tissues compared with normal colorectal tissues. Expression of NGAL was related to the deeper invasion of CRC $(\mathrm{P}=0.026)$, while NGALR was significantly associated with a deeper invasion $(\mathrm{P}=0.018)$ and a high degree of Tumor, Node and Metastasis stages $(\mathrm{P}=0.042)$ in CRC. The NGAL/NGALR co-expression was associated with poor cellular differentiation $(\mathrm{P}=0.004)$. Positive correlations between NGAL and NGALR $(\mathrm{r}=0.432, \mathrm{P}<0.01)$, NGAL and ferritin $(\mathrm{r}=0.374, \mathrm{P}<0.001)$, NGALR and Ki67 $(\mathrm{r}=0.228, \mathrm{P}<0.05)$, NGAL/NGALR co-expression and ferritin $(r=0.349, \mathrm{P}<0.001)$, as well as NGAL/NGALR co-expression and Ki67 ( $\mathrm{r}=0.205$,
\end{abstract}

Correspondence to: Dr En-Min Li, Department of Biochemistry and Molecular Biology, Medical College of Shantou University, No. 22 XinLing Road, Shantou 515041, P.R. China

E-mail:nmli@stu.edu.cn

Dr Li-Yan Xu, Institute of Oncologic Pathology, Medical College of Shantou University, No. 22 XinLing Road, Shantou 515041, P.R. China E-mail: liyanxu1130@yahoo.com.cn

Key words: NGAL, NGALR, colorectal carcinoma, tumour invasion, cell differentiation, immunohistochemistry
$\mathrm{P}<0.05$ ) were observed. However, the expression of NGAL or NGALR was not significantly associated with patient survival. These findings detected an elevated expression of NGAL and NGALR resulting in poor cellular differentiation and a deeper invasion of CRC. Thus, NGALR may be a novel target for the treatment of CRC.

\section{Introduction}

Colorectal carcinoma (CRC) is one of the most common malignancies worldwide. Each year in the United States and Canada, CRC is diagnosed in $>160,000$ people and $\sim 65,000$ succumb to the disease, accounting for at least $10 \%$ of all cancer deaths. The lifetime risk of developing CRC is 1 in 17, affecting men and women alike, with $90 \%$ of cases occurring after the age of 50 years. Of note is that the incidence of CRC has increased substantially in Asia during the past few decades (1).

Neutrophil gelatinase-associated lipocalin (NGAL) is a $25-\mathrm{kDa}$ secreted protein of the lipocalin superfamily (2). An elevated NGAL expression is observed in human cancers such as breast, pancreatic, ovarian and cholesteatoma as well as esophageal squamous cell carcinoma (3-7). Our previous studies demonstrated that the over-expression of NGAL plays an important role in the malignant transformation of human immortalized esophageal epithelial cells and it is involved in the invasion of esophageal squamous cell carcinoma cells (8-10). Others have reported very high expression levels of NGAL in colonic epithelium in areas of inflammation, with a weak expression occasionally seen in some epithelial cells of normal colon (11). However, Lee et al found that the ectopic expression of NGAL in colon cancer cells had little effect on their growth and viability (12). Conflicting results have been reported from various laboratories. Consequently, the clinical importance of NGAL expression in CRC remains unsettled.

Lipocalins are characterized by multiple molecular recognition properties, including the ability to bind to cell surface receptors (13). Several studies found that NGAL binds iron and delivers the latter to cells (14-16). Devireddy et al isolated a specific cell-surface receptor (24p3R/NGALR) for lipocalin $24 \mathrm{p} 3$, a highly conserved murine homolog of NGAL, and demonstrated that the expression of this receptor conferred 
on cells the ability to take up iron or undergo apoptosis depending on the state of $24 \mathrm{p} 3$, independent of the cell type (17). However, the expression patterns and specific features of NGALR in CRC are still unknown. Therefore, this study aimed to investigate the expression of NGAL and NGALR in CRC specimens, and determine any relationship between the expression of these proteins and tumour progression.

\section{Materials and methods}

Cases and clinical parameters. This study was approved by the ethics committee of the Medical College of Shantou University. Written informed consent to use resected samples for research was obtained from patients undergoing surgery.

For this retrospective study, archival formalin-fixed, paraffin-embedded specimens from 102 primary CRC patients were obtained from the Pathology Department of the Medical College of Shantou University, collected between 1992 and 2006.

The patients were 53 males and 49 females (median age 57 years, range 20-81). All patients except one were deceased at the end of follow-up.

Immunohistochemical staining and scoring. Briefly, each tissue section was de-paraffinised, rehydrated and then incubated with fresh $3 \%$ hydrogen peroxide for $10 \mathrm{~min}$. After PBS rinse, antigen retrieval from the tissue was carried out by autoclaving in $0.01 \mathrm{M}$ citrate buffer $(\mathrm{pH} 6.0)$ at $120^{\circ} \mathrm{C}$ for $3 \mathrm{~min}$. Two drops $(100 \mu \mathrm{l})$ or enough to completely cover tissue of the primary antibody were applied to each section and incubated in a moist chamber for $30 \mathrm{~min}$. After the PBS rinse, the tissue sections were incubated for $10 \mathrm{~min}$ at room temperature with HRP polymer conjugate. Subsequently, they were stained with $0.003 \%$ 3,3-diaminobenzidine tetrahydrochloride and $0.005 \%$ hydrogen peroxide in $0.05 \mathrm{M}$ Tris $\bullet \mathrm{HCl}$ (pH 7.2), counterstained with Mayer's hematoxylin, dehydrated and mounted.

Negative controls were prepared by substituting PBS for primary antibody. The polyclonal rabbit NGALR antibody was raised using a C-terminal NGALR peptide (CDHVPLLATPNAL) as the immunogen and then affinitypurified on a peptide-coupled Sepharose column (Beijing Biosynthesis Biotechnology Co., Ltd). This antibody has been selected for its ability to recognize human NGALR. Rat antihuman NGAL antibody (1:50 dilution, R\&D Systems, USA), rabbit anti-human ferritin antibody (1:2000 dilution, Sigma, USA), mouse anti-Ki-67 nuclear antigen (1:100 dilution, Golden Bridge International) and NGALR (1:10 dilution) were used.

The SuperPicTure Polymer Detection and Liquid DAB Substrate kits (Zymed, Carlsbad, CA, USA) were used to carry out immunohistochemical staining. Positive samples were defined as those showing brown signals in the cell cytoplasm or nucleus. When $>5 \%$ of cells in a given specimen were positively stained, it was defined as a positive case.

\section{Statistical analysis}

The association between NGAL or NGALR expression and clinicopathological features of patients was analysed by the
Chi-square or Fisher's exact probability test. The concordance of NGAL and NGALR, NGAL and ferritin, or NGALR and Ki67 expression was determined by the $\kappa$ test. The significance of NGAL or NGALR expression levels to patient survival was examined using the Kaplan-Meier method and log-rank test. Statistical analyses were performed using SPSS for Windows (Version 13.0). The accepted level of significance was $\mathrm{P}<0.05$.

\section{Results}

Overexpression of NGAL is significantly associated with tumour invasion in CRC. Representative results of NGAL immunostaining are shown in Fig. 1. Cytoplasm positivity for NGAL staining was observed in tumour tissues (Fig. 1B), and a weak expression was observed in adjacent normal colon tissues (Fig. 1A). A total of 102 CRC cases were included in the final analysis. NGAL expression was significantly increased in CRC (29/102, 28.4\%) compared with adjacent normal tissues $(0 / 81,0.0 \%)(\mathrm{P}<0.001)$. The percentage of NGAL positivity was significantly associated with $\mathrm{CRC}$ invasion $(\mathrm{P}<0.01)$, and NGAL expression appeared to intensify with the development of the cancer invasion. No significant association was observed between NGAL expression and cell differentiation, although the $\mathrm{P}$-value was borderline $(\mathrm{P}=0.057)$. NGAL expression was not significantly associated with age, gender, cell differentiation, lymph node metastasis or Tumor, Node and Metastasis (TNM) stages ( $\mathrm{P}>0.05$; Table I) in patients with CRC. No significant association between NGAL expression and patient survival was noted $(\mathrm{P}=0.290)$ (Fig. 3).

Overexpression of NGALR is significantly associated with tumour invasion and TNM stages in CRC. NGALR-positive staining was observed in the cytoplasm of CRC tumour cells (Fig. 1D), whereas weak positive staining was observed in a restricted intracellular area close to the cell membrane in adjacent normal tissues (Fig. 1C). The number of CRC specimens staining positive for NGALR $(37 / 102,36.6 \%)$ was significantly higher than that for normal glandular organ (8/93, $8.6 \%)(\mathrm{P}<0.01)$. NGALR expression was strongly associated with deep cancer invasion and with later TNM stages $(\mathrm{P}<0.05$ and $\mathrm{P}<0.05)$. No significant association was found between NGALR expression and gender, age or lymph node metastasis. A significant association was found between tumour TNM stages and patient survival ( $\mathrm{P}=0.043$; Fig. 3). However, there was no significant association between NGALR expression levels and patient survival (Fig. 3).

NGAL and NGALR co-expression is correlated with deeper invasion and tumour progression in patients with CRC. A positive correlation between NGAL and NGALR protein expression was observed in the $102 \mathrm{CRC}$ cases $(\mathrm{r}=0.432$; $\mathrm{P}<0.01$ ). We therefore analyzed the relationship between NGAL and NGALR expression in these cases and the clinicopathological factors. The cases in which NGAL and NGALR were both positive (NGAL/NGALR co-expression) were stratified into group I $(n=19,18.6 \%)$, the positive group, while the rest of the cases were put into group $\Pi(n=83,71.4 \%)$, the negative group. Statistical analysis showed that the two groups exhibited differences in invasion $(\mathrm{P}<0.05)$, cell differentiation 


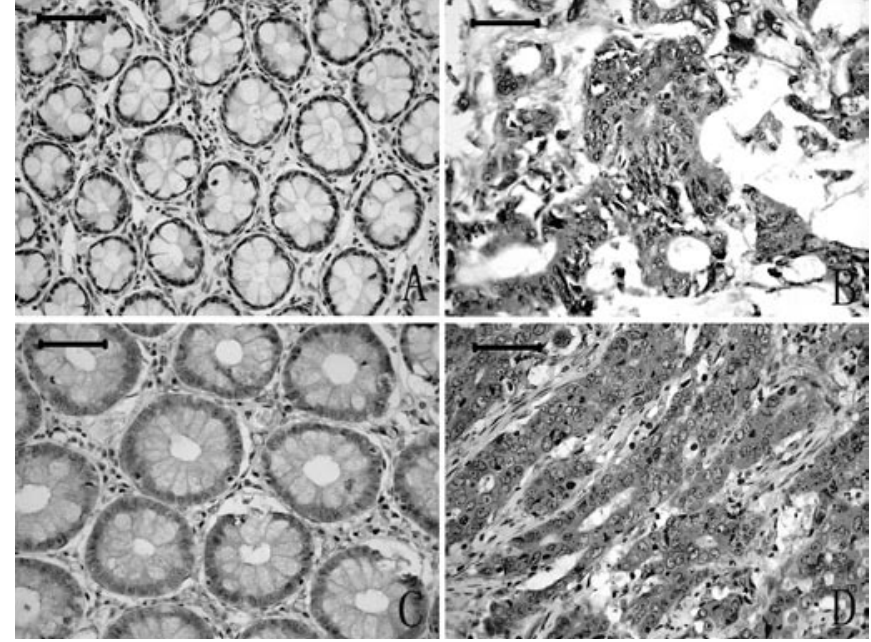

Figure 1. NGAL and NGALR protein expression in CRC (B and D) and adjacent normal glandular organ tissues (A and C). Scale bars, $50 \mu \mathrm{m}$.

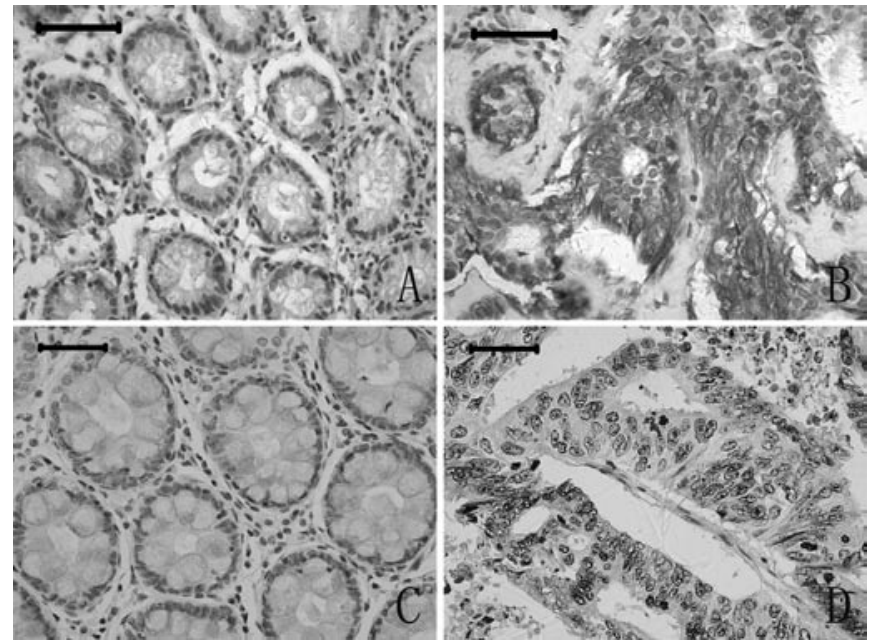

Figure 2. Ferritin and Ki67 protein expression in CRC (B and D) and adjacent normal glandular organ tissues (A and C). Scale bars, $50 \mu \mathrm{m}$.

Table I. Relationship between clinicopathological features and NGALR and/or NGAL positivity.

\begin{tabular}{|c|c|c|c|c|c|c|}
\hline \multirow[t]{2}{*}{ Parameters } & \multicolumn{2}{|c|}{ NGALR } & \multicolumn{2}{|c|}{ NGAL } & \multicolumn{2}{|c|}{ NGAL/NGALR } \\
\hline & $\%$ positive rate & P-value & $\%$ positive rate & P-value & $\%$ positive rate & P-value \\
\hline \multicolumn{7}{|l|}{ Mean age, years (range) } \\
\hline$<57(46)$ & $39.1 \quad(18 / 46)$ & 0.587 & $30.4 \quad(14 / 46)$ & 0.684 & $19.6(9 / 46)$ & 0.825 \\
\hline$\geq 57(56)$ & $33.9(19 / 56)$ & & $26.8(15 / 56)$ & & $17.9(10 / 56)$ & \\
\hline \multicolumn{7}{|l|}{ Gender } \\
\hline Male & $30.2 \quad(16 / 53)$ & 0.184 & $22.4 \quad(11 / 49)$ & 0.198 & $17.0 \quad(9 / 53)$ & 0.675 \\
\hline Female & $42.9 \quad(21 / 49)$ & & $34.0 \quad(18 / 53)$ & & $20.4(10 / 49)$ & \\
\hline \multicolumn{7}{|l|}{ Regional lymph node } \\
\hline NO & $28.8 \quad(15 / 52)$ & 0.112 & $25.0 \quad(13 / 52)$ & 0.593 & $13.5 \quad(7 / 52)$ & 0.172 \\
\hline N1 & $44.0 \quad(22 / 50)$ & & $32.0 \quad(16 / 50)$ & & $24.0(12 / 50)$ & \\
\hline \multicolumn{7}{|l|}{ Primary tumour } \\
\hline $\mathrm{T} 1 / \mathrm{T} 2$ & $10.5 \quad(2 / 19)$ & 0.018 & $10.5 \quad(2 / 19)$ & 0.026 & $5.3 \quad(1 / 19)$ & 0.049 \\
\hline $\mathrm{T} 3$ & $38.3 \quad(23 / 60)$ & & $26.6(16 / 60)$ & & $16.7(10 / 60)$ & \\
\hline $\mathrm{T} 4$ & $52.2(12 / 23)$ & & $47.8 \quad(11 / 23)$ & & $34.8 \quad(8 / 23)$ & \\
\hline \multicolumn{7}{|l|}{ Histopathology } \\
\hline Well-differentiated & $22.7 \quad(5 / 22)$ & 0.193 & $9.1 \quad(2 / 22)$ & 0.057 & $0 \quad(0 / 22)$ & 0.004 \\
\hline Moderately differentiated & $38.0 \quad(27 / 71)$ & & $32.4 \quad(23 / 71)$ & & $21.3(15 / 71)$ & \\
\hline Poorly differentiated & $55.6 \quad(5 / 9)$ & & $44.4 \quad(4 / 9)$ & & $44.4 \quad(4 / 9)$ & \\
\hline \multicolumn{7}{|l|}{ TNM stages } \\
\hline $\mathrm{I} / \Pi \mathrm{a}$ & $23.7 \quad(9 / 38)$ & 0.042 & $23.7 \quad(9 / 38)$ & 0.413 & $7.9 \quad(3 / 38)$ & 0.032 \\
\hline Пb/Ш/IV & $43.6 \quad(28 / 64)$ & & $31.3(20 / 64)$ & & $25.0(16 / 64)$ & \\
\hline Colorectal cancer & $36.3(37 / 102)$ & 0.000 & $28.4(29 / 102)$ & 0.000 & & \\
\hline Normal mucosa & $8.6 \quad(8 / 93)$ & & $0 \quad(0 / 81)$ & & & \\
\hline
\end{tabular}

aPositive, both NGAL and NGALR were positive in one case. Positive rate, positive cases in the proportion of the overall.

$(\mathrm{P}<0.01)$ and TNM stages $(\mathrm{P}<0.05)$. No significant associations with age, gender and patient survival were found for either group (Table I; Fig. 3).
NGAL and NGALR expression is associated with iron transport in cancer cells and increased cell proliferation. To explore whether a high expression of NGAL and NGALR 
Survival Functions

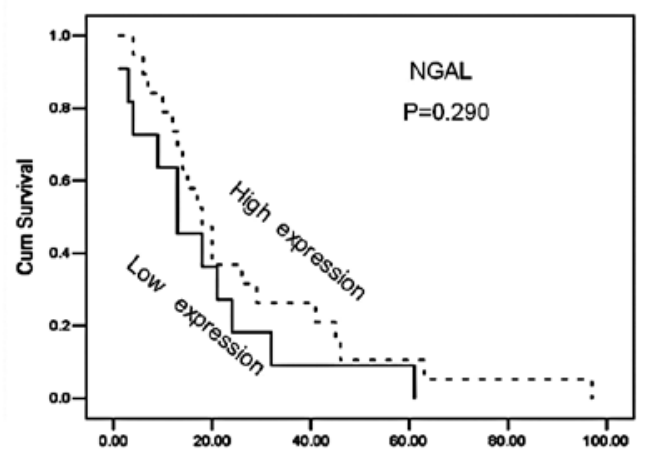

Survival Functions

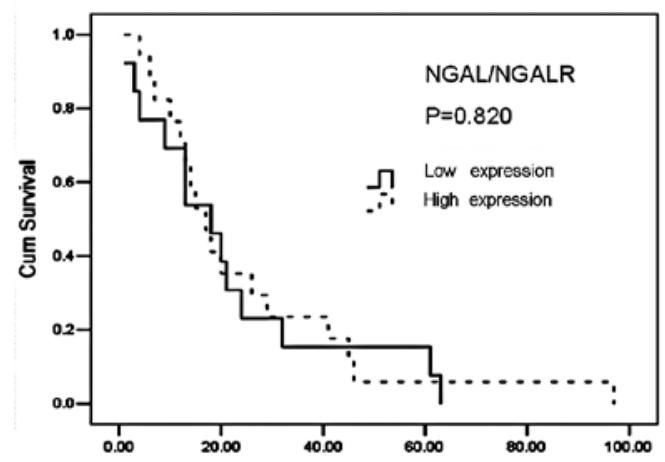

Survival Functions

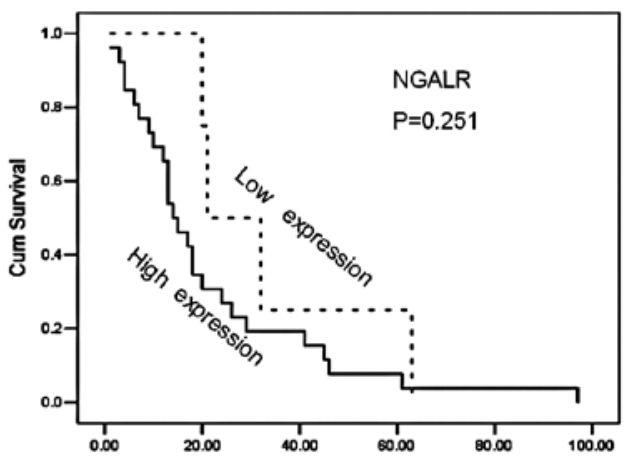

Survival Functions

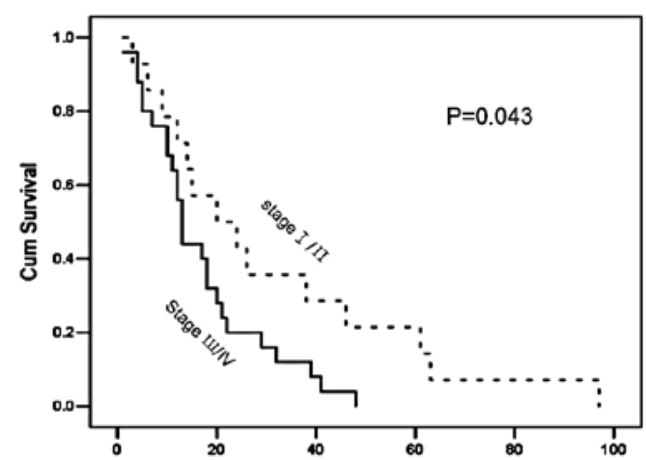

Figure 3. Kaplan-Meier estimates of the overall survival by NGAL, NGALR and NGAL/NGALR and TNM stage status.

is associated with iron transport and cell proliferation, we measured the expression level of ferritin and Ki67 in CRC cells by immunohistochemistry. Ferritin-positive staining was observed in the cytoplasm of tumour and normal cells (Figs. 2A and B). However, of the 102 tumor cases examined, ferritin expression was positive in $40(39.2 \%)$, whereas of the 57 normal specimens examined, ferritin expression was positive in only 2 cases (3.51\%); a statistically significant difference $(\mathrm{P}<0.01)$. Expression of Ki67 was observed in the nucleus of CRC tumour and normal cells (Figs. 2C and D). Of the 102 tumour specimens examined, Ki67 expression was positive in 54 cases (52.9\%), compared to 7 positive specimens in 44 normal cases $(15.9 \%)$; again a significant difference in expression levels $(\mathrm{P}<0.01)$.

As for the relationships among the proteins themselves, there were significant positive associations between the expression of NGAL and ferritin $(\mathrm{r}=0.374, \mathrm{P}<0.001)$, as well as NGALR and Ki67 $(\mathrm{r}=0.228, \mathrm{P}<0.05)$. An analysis of the relationship between NGAL/NGALR co-expression and the expression of ferritin or Ki67 revealed significantly positive correlations for NGAL/NGALR co-expression with ferritin $(\mathrm{r}=0.349, \mathrm{P}<0.001)$ and $\mathrm{Ki67}(\mathrm{r}=0.205 ; \mathrm{P}<0.05)$ in $\mathrm{CRC}$.

\section{Discussion}

NGAL, a member of the lipocalin family, was originally discovered as a protein stored in specific granules of the human neutrophil (2). Our group reported that the over-expression of
NGAL is associated with poor differentiation of oesophageal squamous cell carcinoma (3). In the present study, we found that the expression of NGAL is significantly increased in CRC tissues, and that NGAL/NGALR co-expression is associated with poor cellular differentiation.

A series of studies suggested that NGAL is a novel iron transporter with functions distinct from those of transferrin (16-18). Moreover, a specific cell-surface receptor of $24 \mathrm{p} 3 /$ NGAL (24p3R/NGALR) was cloned (17). Over-expression of $24 \mathrm{p} 3 \mathrm{R} / \mathrm{NGALR}$ in cells induces binding and the uptake of 24p3/NGAL, which results in specific biological responses: iron-loaded 24p3/NGAL increases the intracellular iron concentration without promoting apoptosis, while iron-lacking 24p3/NGAL decreases intracellular iron levels, which induces expression of the proapoptotic protein Bim, leading to apoptosis (17). In the present study, NGALR was observed to be significantly up-regulated in CRC tissues. Furthermore, the co-expression of NGAL and NGALR is associated with CRC development, as is the expression of ferritin and Ki67. A correlation analysis revealed significant positive correlations for NGAL and NGALR, NGAL and ferritin, as well as NGALR and Ki67. These data suggest that NGAL and NGALR participate in intracellular iron transport/accumulation and contribute to the poor differentiation of tumour cells. Results of a large prospective epidemiological study suggest that iron results in an increased risk for CRC (19). High dietary iron promotes the production of reactive oxygen species, which activates the activator protein 1 and nuclear factor $\mathrm{\kappa B}$ signal 
transduction pathways, leading to transcription of the genes involved in cell growth regulation (20-24). We assume that NGAL and NGALR play an important role in CRC through an increase of the iron content of cells, possibly activating some iron-sensitive gene(s) involved in tumour infiltration.

Our previous work showed that NGAL expression is significantly correlated with the depth of tumour invasion in oesophageal squamous cell carcinoma, a pathological process accompanied by over-expression of the NGAL/MMP-9 (matrix metalloproteinase 9) complex (4). MMP-9 is a proteolytic enzyme that degrades the extracellular matrix leading to connective tissue remodelling during normal biological processes and tumour invasion. NGAL directly associates with MMP-9, protecting it from degradation and resulting in increased MMP-9 activity $(14,25,26)$. Interestingly, we found that the levels of NGAL, NGALR and NGAL/NGALR are associated with CRC invasion. A role for NGAL in breast cancer invasion has been suggested following the observation that the NGALoverexpressing human breast cancer cell line MCF-7 exhibits an elevated tumour cell proliferative fraction (27).

Several examples of ligand-receptor interactions involved in tumour progression are known. Relaxin binds to LGR7 (relaxin receptor) and activates signalling cascades, leading to changes in tumour cell proliferation and altered motility (28). Receptor activator of nuclear factor $\kappa \mathrm{B}$ is expressed on prostate cancer cells and promotes invasion in a receptor activator of nuclear factor $\kappa \mathrm{B}$ ligand-dependent manner (29). Koshiba et al reported that the SDF-1/CXCR4 receptor-ligand system may be involved in the progression of pancreatic cancer, participating in tumour cell migration and angiogenesis (30). In light of these discoveries, we speculate that the NGAL/NGALR interaction is a key regulator of tumour growth and invasion in two distinct pathways: one regulating genes which promote cancer invasion, and the other activating iron-related genes also involved in cancer invasion. However, whether the aberrant expression of NGAL and NGALR in these pathways leads to an enhanced invasion of CRC needs to be investigated.

This study is the first to investigate expression of the NGALR protein in a large series of CRC, and to reveal a role of NGAL and NGALR in tumour invasion and cell differentiation in CRC. The most important findings are: i) expression of NGAL, NGALR, ferritin and Ki67 is elevated in CRC; and ii) over-expression of NGAL and NGALR is associated with cell differentiation and tumour invasion in CRC. Thus, our findings suggest that NGAL and NGALR are involved in the transformation and progression of CRC. Therefore, NGALR may be a novel target for the treatment of CRC.

\section{Acknowledgements}

We are very grateful to Professor Ming-Yao Wu and technicians Qiao-Shan Li and Rui-Ming Zheng from the Pathology Department of the Medical College of Shantou University for the specimens. Funding was provided by grants from the National High Technology Research and Development Program of China (No. 2006AA02A403), the National Natural Science Foundation of China (No. 30672376, No. 30772485), the Specialized Research Fund for the Doctoral Program of Higher Education of China (No. 20050560002 and No.
20050560003), and the Guangdong Scientific Fund for Key Items (No. 37788, No. 5104541 and No. 7118419).

\section{References}

1. Sung JJ, Lau JY, Goh KL and Leung WK: Increasing incidence of CRC in Asia: implications for screening. Lancet Oncol 6: 871-876, 2005.

2. Kjeldsen L, Johnsen AH, Sengel $\varnothing v \mathrm{H}$ and Borregaard N: Isolation and primary structure of NGAL, a novel protein associated with human neutrophil gelatinase. J Biol Chem 268: 10425-10432, 1993.

3. Zhang H, Xu L, Xiao D, Xie J, Zeng H, Wang Z, Zhang X, Niu Y, Shen Z, Shen J, Wu X and Li E: Upregulation of neutrophil gelatinase-associated lipocalin in oesophageal squamous cell carcinoma: significant correlation with cell differentiation and tumour invasion. J Clin Pathol 60: 555-561, 2007.

4. Stoesz SP, Friedl A, Haag JD, Lindstrom MJ, Clark GM and Gould MN: Heterogeneous expression of the lipocalin NGAL in primary breast cancers. Int J Cancer 79: 565-572, 1998.

5. Furutani M, Arii S, Mizumoto $M$, Kato $M$ and Imamura $M$ : Identification of a neutrophil gelatinase-associated lipocalin mRNA in human pancreatic cancers using a modified signal sequence trap method. Cancer Lett 122: 209-214, 1998.

6. Bartsch $\mathrm{S}$ and Tschesche $\mathrm{H}$ : Cloning and expression of human neutrophil lipocalin cDNA derived from bone marrow and ovarian cancer cells. FEBS Lett 357: 255-259, 1995.

7. Woo HJ, Park JC, Bae CH, Song SY, Lee HM and Kim YD: Up-regulation of neutrophil gelatinase-associated lipocalin in cholesteatoma. Acta Otolaryngol 21: 1-6, 2008.

8. Xu LY, Li EM, Xiong HQ, Shen ZY and Cai WJ: Study of neutrophil gelatinase-associated lipocalin (NGAL) gene overexpression in the progress of malignant transformation of human immortalized esophageal epithelial cell. Prog Biochem Biophys 28: 839-843, 2001.

9. Li EM, Xu LY, Cai WJ, Xiong HQ, Shen ZY and Zeng Y: Functions of neutrophil gelatinase-associated lipocalin in the esophageal carcinoma cell line SHEEC. Acta Biochim Biophys Sin 35: 247-254, 2003.

10. Lin JL, Xu LY, Li EM, Cai WJ, Niu YD, Fang KY, Xiong HQ, Shen ZY and Zeng Y: Antisense blocking of NGAL gene expression affects the microfilament cytoskeleton in SHEEC esophageal cancer cells. Prog Biochem Biophys 31: 409-415, 2004.

11. Nielsen BS, Borregaard N, Bundgaard JR, Timshel S, Sehested M and Kjeldsen L: Induction of NGAL synthesis in epithelial cells of human colorectal neoplasia and inflammatory bowel diseases. Gut 38: 414-420, 1996.

12. Lee HJ, Lee EK, Lee KJ, Hong SW, Yoon Y and Kim JS: Ectopic expression of neutrophil gelatinase-associated lipocalin suppresses the invasion and liver metastasis of colon cancer cells. Int J Cancer 118: 2490-2497, 2006.

13. Flower DR: Beyond the superfamily: the lipocalin receptors. Biochim Biophys Acta 1482: 327-336, 2000.

14. Goetz DH, Holmes MA, Borregaard N, Bluhm ME, Raymond KN and Strong RK: The neutrophil lipocalin NGAL is a bacteriostatic agent that interferes with siderophore-mediated iron acquisition. Mol Cell 10: 1033-1043, 2002.

15. Yang J, Mori K, Li JY and Barasch J: Iron, lipocalin, and kidney epithelia. Am J Physiol Renal Physiol 285: F9-F18, 2003.

16. Yang J, Goetz D, Li J-Y, Wang W, Mori K, Setlik D and Du T, Erdjument-Bromage H, Tempst P, Strong R and Barasch J: An iron delivery pathway mediated by a lipocalin. Mol Cell 10: 1045-1056, 2002.

17. Devireddy LR, Gazin C, Zhu X and Green MR: A cell-surface receptor for lipocalin $24 \mathrm{p} 3$ selectively mediates apoptosis and iron uptake. Cell 123: 1293-1305, 2005.

18. Mori K, Lee HT, Rapoport D, Drexler IR, Foster K, Yang J, Schmidt-Ott KM, Chen X, Li JY, Weiss S, Mishra J, Cheema FH, Markowitz G, Suganami T, Sawai K, Mukoyama M, Kunis C, D'Agati V, Devarajan P and Barasch J: Endocytic delivery of lipocalin-siderophore-iron complex rescues the kidney from ischemia-reperfusion injury. J Clin Invest 115: 610-621, 2005.

19. Wurzelmann JI, Silver A, Schreinemachers DM, Sandler RS and Everson RB: Iron intake and the risk of colorectal cancer. Cancer Epidemiol Biomarkers Prev 5: 503-507, 1996.

20. Stone WL, Krishnan K, Campbell SE, Qui M, Whaley SG and Yang H: Tocopherols and the treatment of colon cancer. Ann N Y Acad Sci 1031: 223-233, 2004. 
21. Sawa T, Akaike T, Kida K, Fukushima Y, Takagi K and Maeda H: Lipid peroxyl radicals from oxidized oils and heme-iron: implication of a high-fat diet in colon carcinogenesis. Cancer Epidemiol Biomarkers Prev 7: 1007-1012, 1998.

22. Lund EK, Fairweather-Tait SJ, Wharf SG and Johnson IT: Chronic exposure to high levels of dietary iron fortification increases lipid peroxidation in the mucosa of the rat large intestine. J Nutr 131: 2928-2931, 2001.

23. Kuratko CN: Decrease of manganese superoxide dismutase activity in rats fed high levels of iron during colon carcinogenesis. Food Chem Toxicol 36: 819-824, 1998.

24. Valko M, Rhodes CJ, Moncol J, Izakovic M and Mazur M: Free radicals, metals and antioxidants in oxidative stress-induced cancer. Chem Biol Interact 160: 1-40, 2006.

25. Yan L, Borregaard N, Kjeldsen L and Moses MA: The high molecular weight urinary matrix metalloproteinase (MMP) activity is a complex of gelatinase B/MMP-9 and neutrophil gelatinase-associated lipocalin (NGAL). J Biol Chem 276: 37258-37265, 2001.

26. Tschesche H, Zölzer V, Triebel S and Bartsch S: The human neutrophil lipocalin supports the allosteric activation of matrix metalloproteinases. Eur J Biochem 268: 1918-1928, 2001.
27. Fernández CA, Yan L, Louis G, Yang J, Kutok JL and Moses MA: The matrix metalloproteinase-9/neutrophil gelatinase-associated lipocalin complex plays a role in breast tumor growth and is present in the urine of breast cancer patients. Clin Cancer Res 11: 5390-5395, 2005.

28. Klonisch T, Bialek J, Radestock Y, Hoang-Vu C and HombachKlonisch S: Relaxin-like ligand-receptor systems are autocrine/ paracrine effectors in tumor cells and modulate cancer progression and tissue invasiveness. Adv Exp Med Biol 612: 104-118, 2007.

29. Armstrong AP, Miller RE, Jones JC, Zhang J, Keller ET and Dougall WC: RANKL acts directly on RANK-expressing prostate tumor cells and mediates migration and expression of tumor metastasis genes. Prostate 68: 92-104, 2008.

30. Koshiba T, Hosotani R, Miyamoto Y, Ida J, Tsuji S, Nakajima S, Kawaguchi M, Kobayashi H, Doi R, Hori T, Fujii N and Imamura M: Expression of stromal cell-derived factor 1 and CXCR4 ligand receptor system in pancreatic cancer: a possible role for tumor progression. Clin Cancer Res 6: 3530-3535, 2000. 